\title{
All-Optical Pulse Processing for Advanced Photonic Communication System
}

\author{
L.P. Barry, K. Bondarczuk, K. J. Dexter, P.J. Maguire, D.A. Reid \\ J. O'Dowd*, W.H. Guo*, M. Lynch*, A.L. Bradley*, J.F. Donegan* \\ Research Institute for Network and Communications Engineering, DCU, Dublin 9, Ireland \\ * School of Physics, Trinity College Dublin, Dublin 1, Ireland \\ Tel: (3531) 7005883,Fax: (3531) 7005508,e-mail: liam.barry@dcu.ie
}

\begin{abstract}
This paper investigates the use of a Two-Photon Absorption photodetector for high speed processing of ultrashort optical pulses in advanced photonic communication systems. Specifically the paper describes how the Two-Photon Absorption photodetector maybe employed for chromatic dispersion monitoring in high-speed, wavelength division multiplexed networks, and also for reducing multiple access interference noise in an optical code division multiplexed system.

Keywords: dispersion monitoring, wavelength division multiplexing, two-photon absorption, microcavity, optical pulse processing, optical communications, code division multiplexing.

\section{INTRODUCTION}

Due to continued growth of the Internet and the introduction of new broadband services, it is anticipated that high-speed all-optical data processing will be a key enabling technology in future advanced photonic communications systems. These all-optical processing components will need to use nonlinear optical effects, present in fibres, semiconductors and optical crystals, to achieve high-speed operation. A prime candidate is Two-Photon Absorption (TPA) in semiconductors. TPA is a nonlinear optical-to-electrical conversion process where two photons are absorbed simultaneously in the generation of a single electron-hole pair. The generated photocurrent is proportional to the square of the incident optical power falling on the detector, and it is this nonlinear response, combined with TPA's ultra-fast response time that allows TPA to be used for high-speed optical processing. In this paper we demonstrate how a TPA detector maybe employed both for Chromatic Dispersion (CD) monitoring in a high-speed Wavelength Division Multiplexed (WDM) system, and also for the reduction of Multiple Access Interference (MAI) in an optical Code Division Multiplexed (OCDM) system.
\end{abstract}

\section{TPA MICROCAVITY}

In a TPA detector the semiconductor band-gap energy is chosen to be higher than the incident photon energy. Under this condition single photons do not possess sufficient energy to generate an electron-hole pair. Therefore the majority of the photocurrent generated by the detector will result from TPA. This TPA photocurrent is proportional to the square of optical power falling on the detector, and it has an ultrafast response time $\left(10^{-14} \mathrm{fs}\right.$ at $1550 \mathrm{~nm}$ [1]). One of the major problems associated with using the TPA process is the inherent inefficiency associated with the nonlinear process, resulting in the need for high optical intensities typically not found in an optical communications network. One way to overcome this problem is by the adoption of Resonance Cavity Enhancement (RCE) technology within the detector. It has already been shown that by employing RCE technology, the TPA response can be enhanced by over four orders of magnitude when compared to non-cavity devices [2]. One interesting feature of using a RCE-based device is the fact that the incident signal is only enhanced over a narrow wavelength range determined by the device design [2]. This characteristic of the microcavity allows for the processing of a single WDM channel without the necessity of using an additional external optical filter.

\section{MICROCAVITY CHARACTERISATION}

The fabricated TPA microcavity device consists of two GaAs/AlAs Distributed Bragg Reflector (DBR) mirrors surrounding an undoped GaAs active region. The active region is $460 \mathrm{~nm}$ thick with a band-gap energy $1.428 \mathrm{eV}$. The device length is designed to enhance the TPA absorption wavelength within the $1.5 \mu \mathrm{m}$ range. Initial characterisation of the TPA microcavity was carried out using a $10 \mathrm{GHz}, 2$ ps pulse source. Fig. 1(a) shows a plot of the TPA photocurrent generated as a function of incident optical power showing a nonlinear response over 3 orders of magnitude dynamic range. Fig. 1(b) shows the TPA response for varied incidence wavelength. The spectral linewidth is $2.14 \mathrm{~nm}$. As mentioned earlier, one of the advantages of using a RCE design is the fact that the incident signal is only enhanced over a narrow spectral range determined by the microcavity design. It has already been shown that the resonance wavelength of the microcavity can be varied by changing the incident angle [3]. Fig. 1(c) shows a plot of the resonance wavelength of the microcavity as 
a function of varying incident angle $\theta$. From the plot, the resonance wavelength can be tuned by $80 \mathrm{~nm}$, with the device having a $\sin ^{2}(\theta)$ response [3].
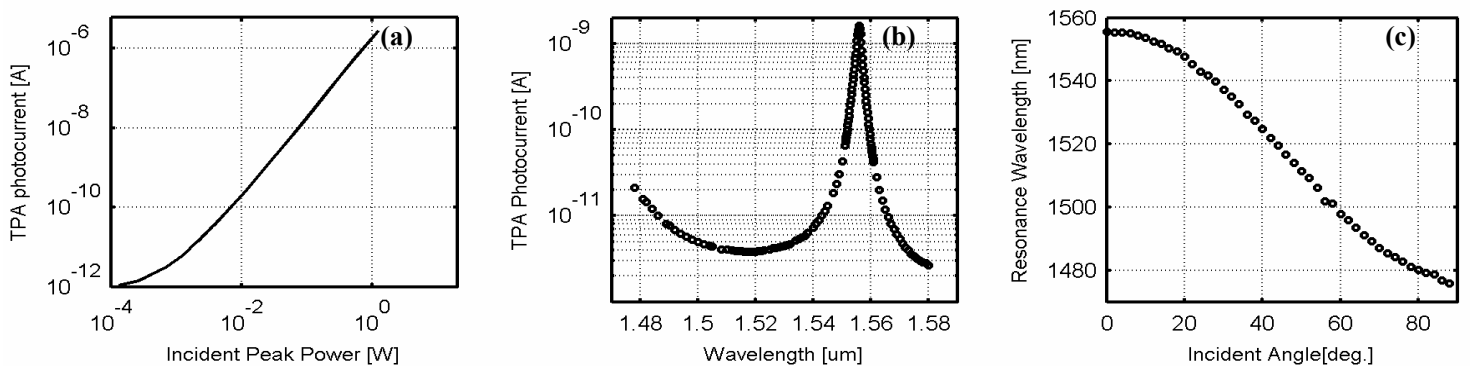

Fig 1 (a) TPA photocurrent for varied incident pulse-signal average power, (b) resonance characteristics for normal incident angle, (c) measured cavity resonance wavelength for varied incidence angle.

\section{WDM CHANNEL CHROMATIC DISPERSION MONITORING AT HIGH BIT DATA RATES}

Fluctuations of $\mathrm{CD}$ in an optical network may occur due to unstable environmental conditions e.g. temperature fluctuations [4], mechanical stress or routing the data signals through different optical paths. These variations of $\mathrm{CD}$ become a serious problem in ultra fast systems operating with rates exceeding $40 \mathrm{Gbps}$. Therefore there is a need to continuously monitor the amount of $\mathrm{CD}$ and compensate it in real time. The TPA effect can be employed for $\mathrm{CD}$ monitoring since its response to optical signals depends on the pulse widths for the same incident average power. According to [5], assuming that the incident optical pulses have a Gaussian shape and the cavity resonance enhancement is given by wavelength dependent function $\eta(\lambda)$, the average photocurrent generated by the TPA microcavity in the absence of SPA by a Pseudo Random Bit Sequence (PRBS) incident optical RZ signal is given by:

$$
i_{\text {ave }}=\frac{e V \beta T \eta(\lambda)}{h \nu \sqrt{\pi} \tau} I_{\text {ave }}^{2}
$$

where $V$ is the volume of the active region of the TPA detector, $\beta$ is the TPA coefficient, $T$ is the period of the incident pulse stream, $\tau$ is the pulse width coefficient $\left(\tau=1.7 \tau_{\mathrm{FWHM}}\right)$ and $I_{\text {ave }}$ is the average intensity of the modulated (PRBS) incident optical signal. The equation is true as long as pulses in adjacent bit slots are not overlapping $(T>>\tau)$. As pulses start spreading into adjacent bit slots due to the effect of $\mathrm{CD}$, eq. (1) is no longer valid, with the resultant output from the TPA detector tending toward a constant value:

$$
i_{\text {const }}=\frac{e V \beta \eta(\lambda)}{2 h v} I_{\text {ave }}^{2}
$$

The experimental work undertaken involves CD monitoring of two wavelength channels, each operating at an aggregate data rate of $160 \mathrm{~Gb} / \mathrm{s}$. The experimental setup is shown in Fig. 2. The pulse sources used generate $10 \mathrm{GHz}$ pulse trains with duration of around $2 \mathrm{ps}$. The wavelength channels were separated spectrally by $1.25 \mathrm{THz}(10 \mathrm{~nm})$, operating at frequencies of $192.7 \mathrm{THz}(1556 \mathrm{~nm})$ and $193.9 \mathrm{THz}(1546 \mathrm{~nm})$.

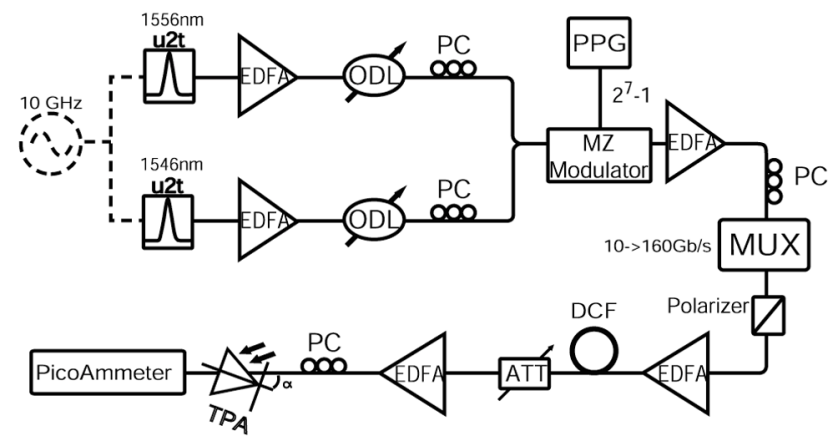

Fig.2 Experiment setup for sequential CD monitoring of two wavelength channels using a TPA microcavity

Both channels were amplified and combined together using a $3 \mathrm{~dB}$ passive fibre coupler before passing through an optical modulator driven with $2^{7}-1$ RZ PRBS data pattern. Both channels were then passed through an OTDM multiplexer. The WDM signal was then passed through different lengths of Dispersion Compensation Fibre (DCF). The generated photocurrent for the various DCF lengths and repetition rates was measured on the PicoAmmeter. Initially, the cavity was set at normal incidence to monitor CD in the fist channel. Then the cavity was tilted by $22^{\circ}$ (to shift the cavity resonance wavelength by $10 \mathrm{~nm}$ ) to monitor the second channel. The measured TPA photocurrents versus the introduced accumulated dispersion for both wavelength channels 
are displayed in Fig. 3. The measurements were carried out for $10 \mathrm{~Gb} / \mathrm{s}, 40 \mathrm{~Gb} / \mathrm{s}$ and $160 \mathrm{~Gb} / \mathrm{s}$. The solid lines are simulation results obtained using a basic model, assuming incoherent pulse overlapping. Excellent agreement is shown indicating the potential of a TPA based CD monitor.
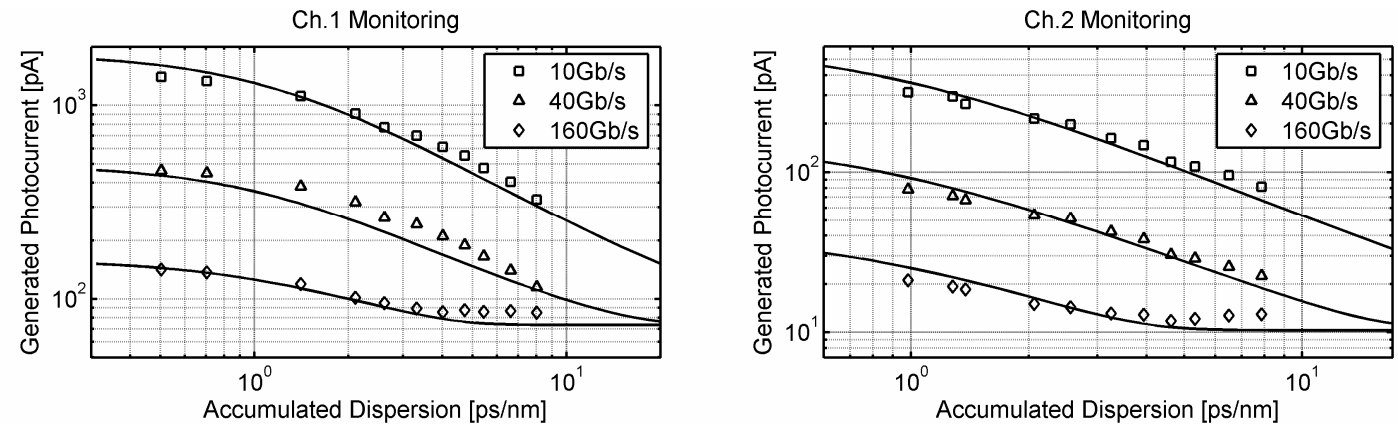

Fig. 3 Sequential CD monitoring: (a) Channel 1 operating at $1556 \mathrm{~nm}$; (b) Channel 2operating at 154 6nm. Points correspond to the experimental results for various repetition rates while the solid lines are the simulation curves assuming pulses incoherent overlapping and finite cavity bandwidth.

\section{MAI NOISE REDUCTION IN OCDMA SYSTEM}

While current multiplexing techniques (WDM and OTDM) are highly suited to long-haul networks, their requirements of high precision wavelength-stabilization techniques and strict synchronization control limit their overall flexibility and scalability in Local Area Networks (LAN's) and Fibre to the Home (FTTH) networks [6]. In comparison, Optical Code Division Multiple Access OCDMA provides several unique advantages such as asynchronous transmission, soft capacity on demand, and quality of service control [7]. While a number of different methods for encoding and decoding optical signals have been presented in OCDMA literature [8], each coding technique suffers from the phenomenon of Multiple Access Interference (MAI). MAI is generated when cross-correlation noise generated from the improper decoding of an optical signal is incident on an optical receiver. This noise increases proportionally with the number of users transmitting data across the network, limiting the overall system performance. Optical time gating and optical thresholding have been suggested in order to suppress MAI [9], however, the methods described in the literature have various limitations. In this work we show how a TPA detector may reduce the level of MAI interference in an OCDMA system.

The nonlinear response of a TPA detector is determined by its Single Photon Absorption (SPA) coefficient and Two Photon Absorption (TPA) coefficient, $\alpha$ and $\beta$ respectively. The equations governing the overall contributions of SPA and TPA to the total absorption of incident light are given in [10]. Using a $1.3 \mu \mathrm{m}$ FP laser as a TPA detector, the TPA photocurrent generated as a function of incident optical peak power was experimentally characterised and is shown in Fig. 4(a). Using a parameter-fitting algorithm the $\alpha$ and $\beta$ coefficients for the TPA detector were found to be $1.36 \cdot 10^{-4} \mathrm{~m}^{-1}$ and $2.35 \cdot 10^{-16} \mathrm{~m} / \mathrm{W}$. A simulation model of the TPA detector was constructed using these parameters and the equations in [10]. The nonlinear response of the simulation model is shown in Fig. 4(a) by the dashed line. It can be seen that there is excellent agreement between the response of the physical device and the simulation model.
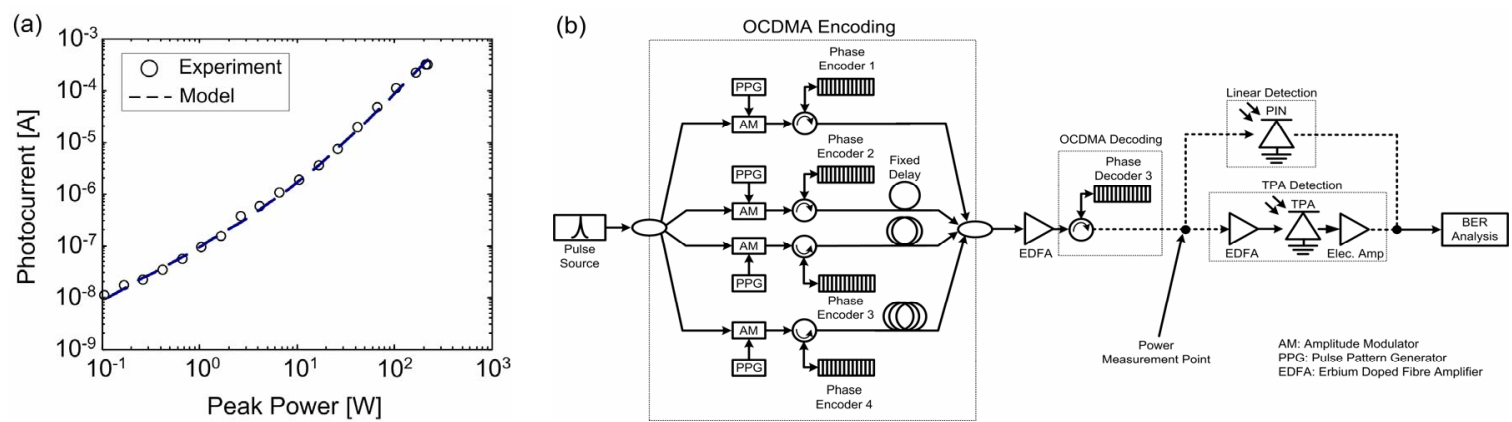

Fig. 4(a) Plot of average TPA photocurrent generated as a function of incident peak power. (b) Model of four channel OCDMA system employing 8-chip temporal phase coding with standard linear and TPA detection methods.

To directly compare the performance of a standard linear detector and the TPA detector, a simulation model of a four channel OCDMA system was created. The simulation model is shown in Fig. 4(b). A $155 \mathrm{MHz}$ optical pulse train with a pulsewidth of 2 ps was split into four copies that were each modulated by four separate PRBS signals. These signals were encoded using 8 chip bipolar temporal phase codes. The aggregate signal is amplified before passing through a decoder matched to one of the channels. The decoded signal is then either 
incident on a standard linear detector or the TPA model. It should be noted that due to the inefficiency of the TPA process, an additional EDFA was used before the TPA detector. The optical power incident on the device is measured before the second EDFA (Fig. 4(b)).

(a)

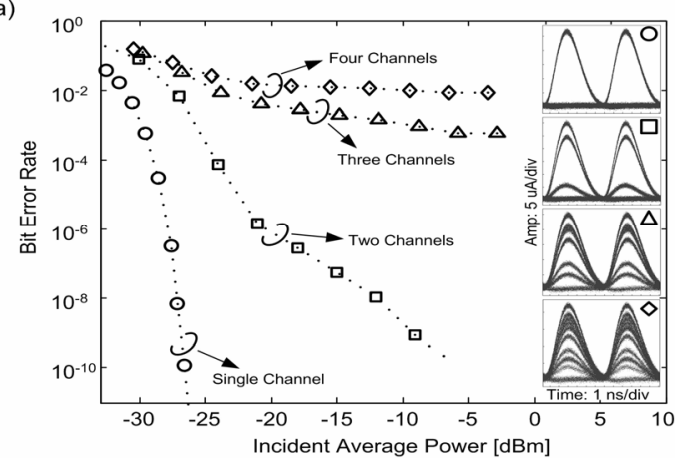

(b)

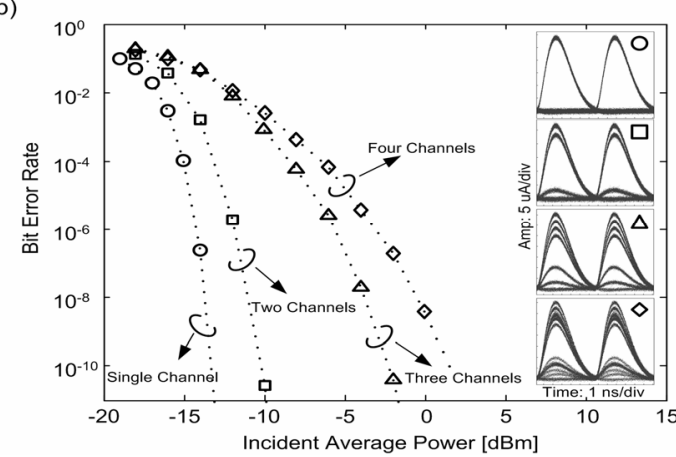

Fig. 5(a) Bit error rate plot for decoder 3 using linear detection as each additional transmitter is added to the system. (b) Bit error rate plot for decoder 3 using TPA detection as each additional transmitter is added to the system. The insets are the electrical eye diagrams obtained for the respective number of transmitting channels.

Fig. 5(a) shows the Bit Error Rate (BER) plot as a function of incident average power for decoder 3 using a linear detector. It can be seen that error free performance (BER of $1 \cdot 10^{-9}$ ) is obtainable for the single user and two user situations, however, the addition of the second user incurs a power penalty of $\sim 16 \mathrm{~dB}$. The further addition of the third and fourth channels to the system introduces an error floor of $\sim 1 \cdot 10^{-3}$. The inset eye diagrams clearly show the degradation of the eye opening as a result of the MAI introduced by the three interfering channels. Fig. 5(b) shows the BER plot obtained for the same decoder using TPA detection. It can be seen that there is $\sim 4 \mathrm{~B}$ power penalty at an error rate of $1 \cdot 10^{-9}$ as a second transmitting channel is added. The addition of the third and fourth channels incurs further penalties of 8 and $3 \mathrm{~dB}$ respectively. This power penalty could be reduced by increasing the code length with respect to the number of channels at the cost of increased transmitter/receiver complexity. Despite the significant power penalty, error-free performance can be achieved using TPA detection in comparison to linear detection.

\section{CONCLUSIONS}

In this paper we have investigated the use of a Two-Photon Absorption (TPA) photodetector for high speed processing of ultra-short optical pulses in advanced photonic communication systems We have demonstrated the use of TPA for both CD monitoring of high-speed WDM data signals at rates of up to $160 \mathrm{~Gb} / \mathrm{s}$, and for the reduction of MAI noise in an OCDMA system. Future work will look at optimising the TPA detector in order to maximise performance in specific applications.

\section{REFERENCES}

[1] J. Donegan: Two-photon absorption speeds optical switching, Lightw. Europe, vol. 1, pp. 31, 2002.

[2] H. Folliot, et al.: Two-photon absorption photocurrent enhancement in bulk AlGaAs semiconductor microcavities, Appl. Phys. Lett, vol. 80, no. 8, pp. 1328-1330, 2002.

[3] T. Krug, et al.: Resonance Tuning of Two-Photon Absorption Microcavities, IEEE Photon. Technol. Lett, vol. 18, no. 2, pp. 433-435, Jan. 2006.

[4] W.H. Hatton and M. Nishimura: Temperature dependence of chromatic dispersion in single mode fibers, J. Lightwave Technol., vol. 4, no. 10, pp. 1552-1555, 1986.

[5] Z. Zheng, et al.: Ultrafast optical thresholding based on two-photon absorption GaAs Waveguide Photodetectors, IEEE Photon. Technol. Lett, vol. 9, no. 4, pp. 493-495, Apr. 1997.

[6] N. Wada and K. Kitayama: A $10 \mathrm{~Gb} / \mathrm{s}$ Optical Code Division Multiplexing Using 8-Chip Optical Bipolar Code and Coherent Detection, J. Lightwave Technol., vol. 17, no. 10, pp. 1758-1765, 1999.

[7] X. Wang and K. Kitayama: Analysis of Beat Noise in Coherent and Incoherent Time-Spreading OCDMA, J. Lightwave Technol., vol. 22, no. 10, pp. 2226-2235, 2004.

[8] P. C. Teh, et al:: A Comparative Study of the Performance of Seven- 63-chip Optical Code-Division Multiple-Access Encoders and Decoders, J. Lightw. Technol., vol. 19, no. 9, pp. 1352-1365, Sept. 2001.

[9] J. H. Lee, et al:: Reduction of Interchannel Interference Noise in a Two-Channel OCDMA System using a Nonlinear Optical Loop Mirror, IEEE Photon. Technol. Lett., vol. 13, no. 5, pp. 529-531, 2001.

[10] H. Folliot, et al.: Two-Photon-Induced Photoconductivity Enhancement in Semiconductor Microcavities: A Theoretical Investigation, J. Opt. Soc. A. B, vol. 19, no. 10, pp. 2396-2402, 2002. 